\title{
Euler Characteristics of Theta Divisors of Jacobians for Spectral Curves.
}

\author{
A. Nakayashiki ${ }^{a}$ and F.A. Smirnov ${ }^{b}$ O \\ ${ }^{a}$ Faculty of Mathematics, Kyushu University \\ Ropponmatsu 4-2-1, Fukuoka 810-8560, Japan \\ ${ }^{b}$ Laboratoire de Physique Théorique et Hautes Energies 1 \\ Université Pierre et Marie Curie, Tour $161^{\text {er }}$ étage, 4 place Jussieu \\ 75252 Paris cedex 05-France
}

\begin{abstract}
We show how to calculate the Euler characteristic of an affine Jacobi variety of a spectral curve from its defining equations.
\end{abstract}

\footnotetext{
${ }^{0}$ Membre du CNRS

${ }^{1}$ Laboratoire associé au CNRS.
} 


\section{Introduction}

Consider an algebraic curve $X$, its Jacobian $J(X)$ and the theta divisor $\Theta \subset J(X)$. The non-compact variety $J(X)-\Theta$ is known to be an affine algebraic variety, which we call the affine Jacobian variety of $X$.

In the paper [1] we consider, following [2], the algebraic model of an affine hyperelliptic Jacobian variety. Under certain strong assumptions we calculated the topological Euler characteristic and dimensions of singular cohomology groups of it. More precisely we made a conjecture on the explicit forms of cohomologies as the irreducible representations of the symplectic group. Later one of the authors [3] was able to prove these conjectures. In this proof certain very particular properties of hyperelliptic curves were used.

The algebraic model is available for the affine Jacobians of more general algebraic curves of the form:

$$
w^{N}+t_{1}(z) w^{N-1}+\cdots+t_{N}(z)=0
$$

where $\operatorname{deg}\left(t_{j}\right) \leq n j-1$ for some $n$ and the leading coefficient of $t_{N}$ does not vanish (the corresponding construction is explained in detail in [4, 5]). However, the cohomologies of the affine Jacobians of these curves seem much more complicated than in the hyperelliptic case. Up to now we were not able to conjecture their explicit form. Thus even a partial information about those cohomologies is important.

In this paper we shall show that under a certain assumption (Assumption 1 in section 3) the topological Euler characteristic $\chi(J(X)-\Theta)$ of the affine Jacobian of a curve $X$ from (11) can be calculated. It then gives the Euler characteristic of the theta divisor $\Theta$ by the relation:

$$
\chi(\Theta)=-\chi(J(X)-\Theta) .
$$

The paper is organized as follows. In the second section we present a rather abstract scheme into which the algebraic models of affine Jacobians can be put. We consider a certain family of affine algebraic sets parametrized by points of a complex affine space. In the third section we consider the special case when the parameter takes the origin. It is the only case when the affine ring becomes graded. In this case the Euler characteristic can be easily determined in terms of the character of the affine ring. We define a double complex which permits to identify the dimensions of cohomologies in question with the dimensions of the spaces of cochains in the minimal free resolution of the affine ring. In the fourth section we show that the Euler characteristic of the variety at a generic point in the parameter space coincides with the Euler characteristic of the variety at the origin by considering certain deformation of the previous construction. This is the main result of this paper. We apply this result to the family of the affine Jacobians of spectral curves (1) studied in [5] and get the formula for $\chi(\Theta)$. In the last section we shall show that our formula for $\chi(\Theta)$ produces correct values in the case of genus 3 and 4 . The appendix contains a proof of the proposition given in section two.

\section{General setting}

Consider the affine space $\mathbb{C}^{m}$ with coordinates $\left(a_{1}, \cdots, a_{m}\right)$. Consider $m-g$ polynomials $f_{j}\left(a_{1}, \cdots, a_{m}\right)$ and the affine algebraic set $\mathcal{J}_{f^{0}}$ defined by the equations

$$
\begin{aligned}
& f_{1}\left(a_{1}, \cdots, a_{m}\right)=f_{1}^{0}, \\
& \ldots \\
& f_{n-g}\left(a_{1}, \cdots, a_{m}\right)=f_{m-g}^{0},
\end{aligned}
$$

where $f_{j}^{0}$ are complex numbers. We assume that $\mathcal{J}_{0}=\left\{p \in \mathbb{C}^{m} \mid f_{j}(p)=0 \forall j\right\}$ is $g$ dimensional, that is, the maximum of the dimensions of irreducible components is $g$. In the case of the family of the affine Jacobians of spectral curves (11) this assumption is verified by using the explicit parametrization of $\mathcal{J}_{0}$ 䏤, 国. We assign a positive degree $\operatorname{deg}\left(a_{j}\right)$ to each $a_{j}$ and assume that the polynomial $f_{j}$ is homogeneous of degree $\operatorname{deg}\left(f_{j}\right)$ for all $j$. We shall consider several rings:

$$
\begin{aligned}
& A=\mathbb{C}\left[a_{1}, \cdots, a_{m}\right], \quad F=\mathbb{C}\left[F_{1}, \cdots, F_{m-g}\right], \\
& A_{0}=A / \sum\left(f_{j} A\right), \quad A_{f^{0}}=A / \sum\left(\left(f_{j}-f_{j}^{0}\right) A\right) .
\end{aligned}
$$


The rings $F$ and $A$ are polynomial rings of $m$ and $m-g$ variables respectively. The ring $F$ acts on $A$ by the multiplication of $F_{j}=f_{j}$. We define $\operatorname{deg}\left(F_{j}\right)=\operatorname{deg}\left(f_{j}\right)$. Then $A, F$ and $A_{0}$ become graded rings. But this is not the case for $A_{f^{0}}$ if some $f_{j}^{0}$ is not zero. The character of a graded vector space is defined as the generating function of the dimensions of homogeneous subspaces. It is obvious that the characters of $A$ and $F$ are given by

$$
\operatorname{ch}(A)=\prod_{j=1}^{m} \frac{1}{1-q^{\operatorname{deg}\left(a_{j}\right)}}, \quad \operatorname{ch}(F)=\prod_{j=1}^{m-g} \frac{1}{1-q^{\operatorname{deg}\left(f_{j}\right)}} .
$$

The determination of the character of $A_{0}$ is more involved. But the result is simple.

Proposition 1. The character of $A_{0}$ is given by

$$
\operatorname{ch}\left(A_{0}\right)=\frac{\operatorname{ch}(A)}{\operatorname{ch}(F)}
$$

The proof of this proposition is given in Appendix A. For the case of the family of affine hyperelliptic Jacobians, this proposition were proved in [1] by determining the $\mathbb{C}$-basis of $A_{0}$ explicitly. In a similar way to Proposition 2 in 11. we have the following corollary of this proposition.

Corollary 1. As a graded $F$ module, $A$ is a free module: $A \simeq F \otimes_{\mathbb{C}} A_{0}$.

An important feature, which is special for the case related with the family of affine Jacobians, is that on $A$ one can define the action of $g$ commuting vector fields $D_{1}, \cdots, D_{g}$. Thus we assume that there exist algebraically independent $g$ commuting vector fields $D_{1}, \cdots, D_{g}$ acting on $A$ such that

$$
D_{j} f_{k}=0 \quad \forall j, k .
$$

Moreover, we assume that, to every $D_{j}$ a positive degree is prescribed such that

$$
\operatorname{deg}\left(D_{j} x\right)=\operatorname{deg}\left(D_{j}\right)+\operatorname{deg}(x)
$$

for every homogeneous $x \in A$. Then the ring $\mathcal{D}=\mathbb{C}\left[D_{1}, \cdots, D_{g}\right]$ becomes a graded ring and its character is given by

$$
\operatorname{ch}(\mathcal{D})=\prod_{j=1}^{g} \frac{1}{1-q^{\operatorname{deg}\left(D_{j}\right)}} .
$$

By the condition (3) the action of $\mathcal{D}$ on $A$ descends to $A_{0}$ and $A_{f^{0}}$.

Let us introduce differentials $d z_{j}$ dual to the vector fields $D_{j}$. We set $V=\oplus_{j=1}^{g} \mathbb{C} d z_{j}$. We define the spaces of cochains by

$$
C^{k}=A \otimes \wedge^{k} V, \quad C_{0}^{k}=A_{0} \otimes \wedge^{k} V, \quad C_{f^{0}}^{k}=A_{f^{0}} \otimes \wedge^{k} V .
$$

The differential $d=\sum D_{j} \otimes d z_{j}$ defines complexes $\left(C^{\cdot}, d\right),\left(C_{0}^{\cdot}, d\right),\left(C_{f^{0}}, d\right)$ which we shall study. We $\operatorname{set} \operatorname{deg}\left(d z_{j}\right)=$ $-\operatorname{deg}\left(D_{j}\right)$ in order that $\operatorname{deg}(d)=0$. Then $C^{k}$ and $C_{0}^{k}$ become graded vector spaces.

\section{Cohomologies at the origin}

Consider the complex:

$$
0 \longrightarrow C_{0}^{0} \stackrel{d}{\longrightarrow} C_{0}^{1} \stackrel{d}{\longrightarrow} \cdots \stackrel{d}{\longrightarrow} C_{0}^{g-1} \stackrel{d}{\longrightarrow} C_{0}^{g} \stackrel{d}{\longrightarrow} 0 .
$$

This complex is graded. One easily finds its $q$-Euler characteristic [1]:

$$
\chi_{q}\left(C_{0}\right)=\sum_{k=0}^{g}(-1)^{k} \operatorname{ch}\left(C_{0}^{k}\right)=(-1)^{g} q^{-\sum \operatorname{deg}\left(D_{j}\right)} \frac{\operatorname{ch}\left(A_{0}\right)}{\operatorname{ch}(\mathcal{D})} .
$$


Taking the limit $q \rightarrow 1$ we find the Euler characteristic as a number:

$$
\chi\left(C_{0}^{\cdot}\right)=(-1)^{g} \frac{\prod_{j=1}^{m-g} \operatorname{deg}\left(f_{j}\right) \prod_{j=1}^{g} \operatorname{deg}\left(D_{j}\right)}{\prod_{j=1}^{m} \operatorname{deg}\left(a_{j}\right)} .
$$

The fact that the Euler characteristic is finite does not mean that the cohomologies are finite dimensional. However we shall adopt the following assumption:

Assumption 1. $\operatorname{dim} H^{g}\left(C_{0}^{\cdot}\right)<\infty$.

Unfortunately, we do not know how to prove this assumption even in the case of the family of affine Jacobians of (1).

The Assumption 1 together with simple grading arguments guarantee that $A_{0}$ is a finitely generated $\mathcal{D}$-module. Then it has the minimal $\mathcal{D}$ free resolution $(c f$. [6] $\S 19)$ of the form

$$
0 \longrightarrow \mathcal{D}^{b_{0}} \stackrel{\varphi_{0}^{0}(D)}{\longrightarrow} \cdots \longrightarrow \mathcal{D}^{b_{g-2}} \stackrel{\varphi_{0}^{g-2}(D)}{\longrightarrow} \mathcal{D}^{b_{g-1}} \stackrel{\varphi_{0}^{g-1}(D)}{\longrightarrow} \mathcal{D}^{b_{g}} \longrightarrow A_{0} \longrightarrow 0 .
$$

Here minimality condition is $\varphi_{0}^{k}(0)=0$ for all $k$. Consider another complex, the Koszul cpmplex of $D_{1}, \ldots, D_{g}$ :

$$
0 \longrightarrow \mathcal{D} \stackrel{d}{\longrightarrow} \cdots \longrightarrow \mathcal{D} \otimes \wedge^{g-2} V \stackrel{d}{\longrightarrow} \mathcal{D} \otimes \wedge^{g-1} V \stackrel{d}{\longrightarrow} \mathcal{D} \otimes \wedge^{g} V \longrightarrow \mathbb{C} \longrightarrow 0
$$

where $d$ is given by the same formula as before $d=\sum D_{j} \otimes d z_{j}$. This complex is exact. For any non-negative integer $b$, tensoring $\mathcal{D}^{b}$ (over $\mathcal{D}$ ) to (1), we get the exact sequence

$$
0 \longrightarrow \mathcal{D}^{b} \stackrel{d}{\longrightarrow} \cdots \longrightarrow \mathcal{D}^{b} \otimes \wedge^{g-2} V \stackrel{d}{\longrightarrow} \mathcal{D}^{b} \otimes \wedge^{g-1} V \stackrel{d}{\longrightarrow} \mathcal{D}^{b} \otimes \wedge^{g} V \longrightarrow \mathbb{C}^{b} \longrightarrow 0 .
$$

Now we can consider the double complex

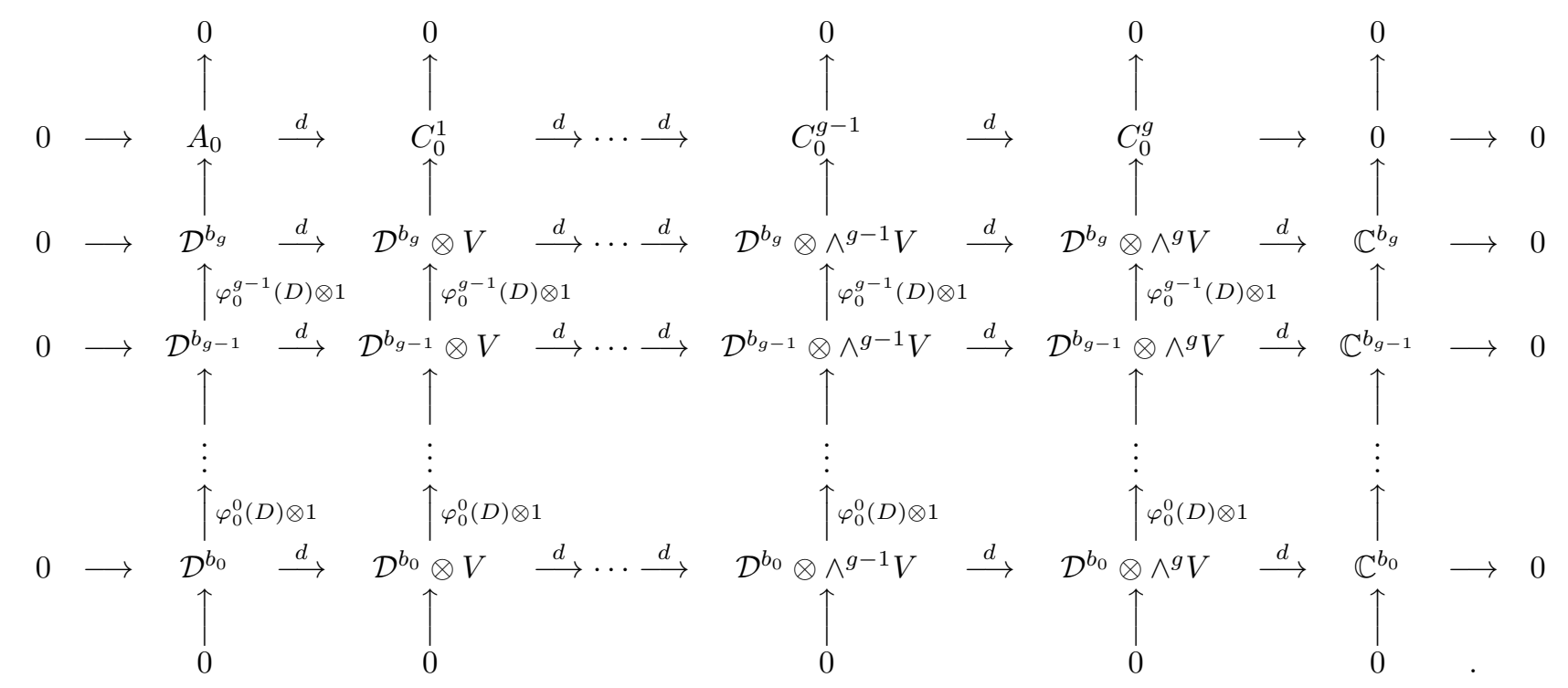

In this double complex each column except the last one is exact, each row except the first one is exact. The minimality condition means that maps of the last column $\mathbb{C}^{b_{k}} \longrightarrow \mathbb{C}^{b_{k+1}}, k \leq g-1$ are all zero maps. Using this diagram one can easily prove that

$$
H^{k}\left(C_{0}^{\cdot}\right) \simeq \mathbb{C}^{b_{k}}
$$




\section{Cohomologies at a generic point}

We set $\mathcal{D}_{F}=F \otimes_{\mathbb{C}} \mathcal{D}$. Under Assumption 1, using Proposition 1, we can prove that $A$ is a finitely generated $\mathcal{D}_{F}$ module in a similar manner to Proposition 4 in [1]. By the uniqueness of the minimal resolution ( $c f$. [6], the graded version of Theorem 20.2), (6) implies the existence of the minimal $\mathcal{D}_{F}$-free resolution of $A$ of the form

$$
0 \longrightarrow \mathcal{D}_{F}^{b_{0}} \stackrel{\varphi^{0}(D)}{\longrightarrow} \cdots \longrightarrow \mathcal{D}_{F}^{b_{g-2}} \stackrel{\varphi^{g-2}(D)}{\longrightarrow} \mathcal{D}_{F}^{b_{g-1}} \stackrel{\varphi^{g-1}(D)}{\longrightarrow} \mathcal{D}_{F}^{b_{g}} \longrightarrow A \longrightarrow 0,
$$

such that $\left.\varphi^{k}(D)\right|_{f_{j}=0}=\varphi_{0}^{k}(D)$. The minimality condition is that $\left.\varphi^{k}(D)\right|_{f_{j}=0, D_{j}=0}=0$. Again consider the double complex:

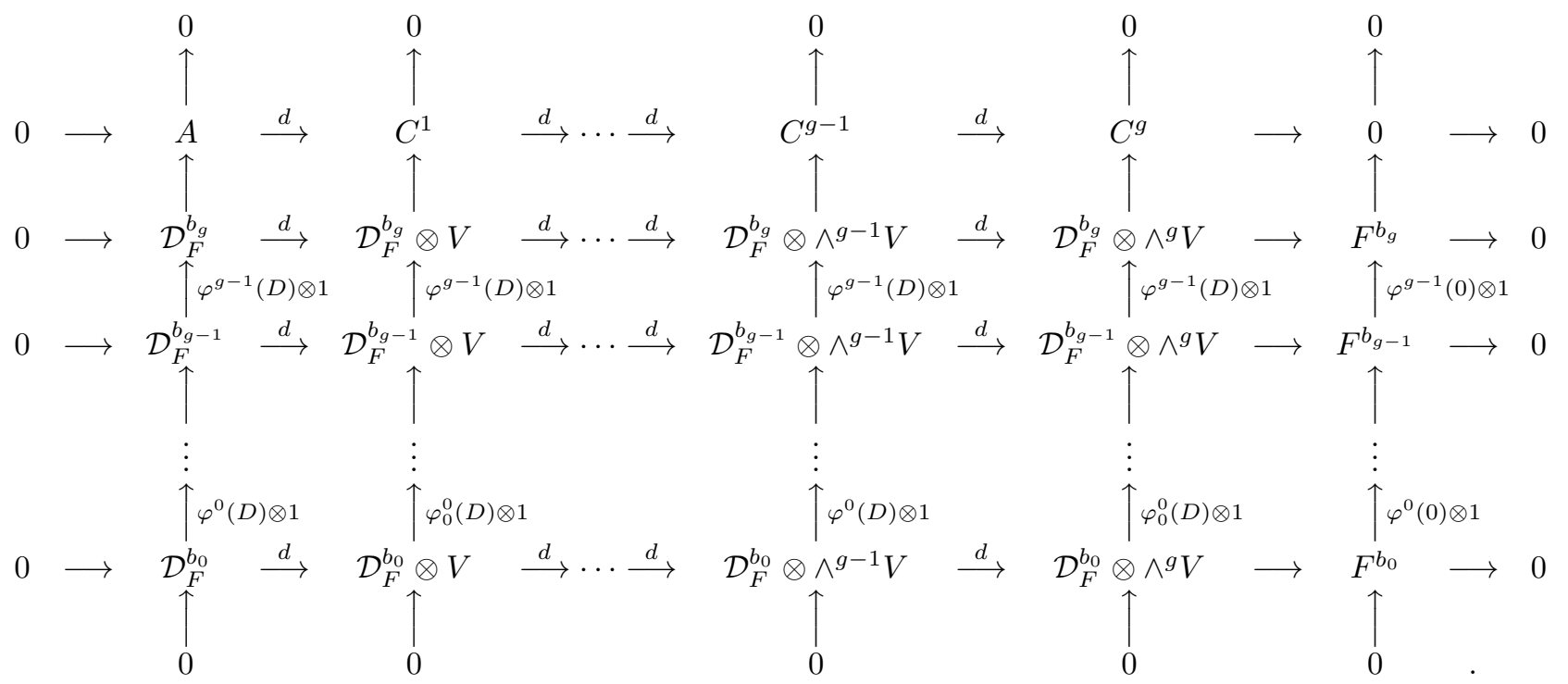

In the diagram each column except the last one and each row except the first one are exact. Since every map in the diagram is $F$-linear and every object in the diagram is an $F$-free module, by tensoring $\mathbb{C}=F / \sum\left(f_{j}-f_{j}^{0}\right) F$ over $F$, we get the double complex:

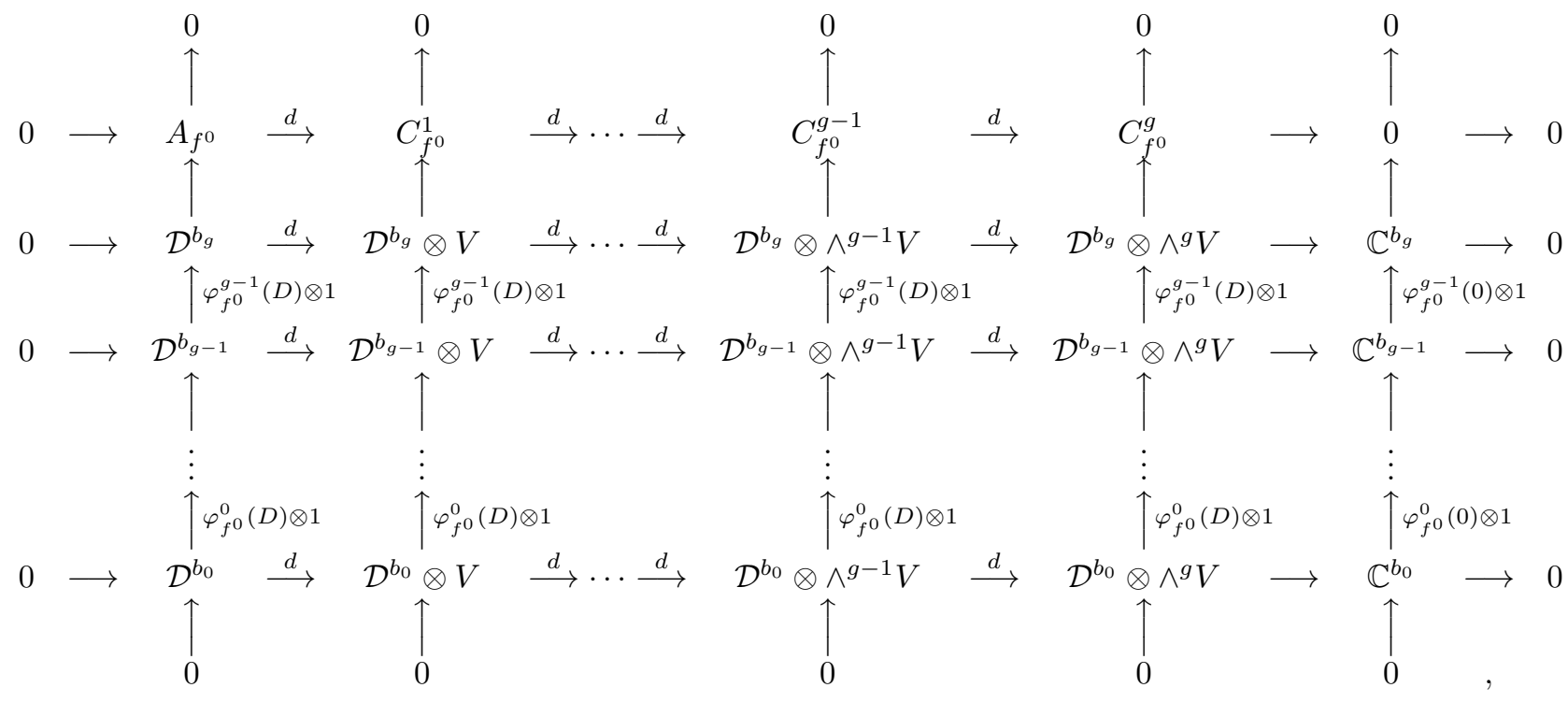

where

$$
\varphi_{f^{0}}^{k}(D)=\left.\varphi^{k}(D)\right|_{f_{j}=f_{j}^{0}}
$$


In the diagram each column except the last one and each row except the first one are exact. One can easily prove the following isomorphism from this diagram:

$$
H^{k}\left(C_{f^{0}}\right) \simeq \operatorname{Ker}\left(\varphi_{f^{0}}^{k}(0) \otimes 1\right) / \operatorname{Im}\left(\varphi_{f^{0}}^{k-1}(0) \otimes 1\right), \quad 1 \leq k \leq g,
$$

where we set $\varphi_{f^{0}}^{g}(0)=0$. It follows that

$$
\begin{aligned}
\chi\left(C_{f^{0}}\right) & =\sum_{k=0}^{g}(-1)^{k}\left(\operatorname{dim}\left(\operatorname{Ker}\left(\varphi_{f^{0}}^{k}(0) \otimes 1\right)\right)-\operatorname{dim}\left(\operatorname{Im}\left(\varphi_{f^{0}}^{k-1}(0) \otimes 1\right)\right)\right) \\
& =\sum_{k=0}^{g}(-1)^{k}\left(\operatorname{dim}\left(\operatorname{Ker}\left(\varphi_{f^{0}}^{k}(0) \otimes 1\right)\right)+\operatorname{dim}\left(\operatorname{Im}\left(\varphi_{f^{0}}^{k}(0) \otimes 1\right)\right)\right) \\
& =\sum_{k=0}^{g}(-1)^{k} b_{k}=\chi\left(C_{0}^{\cdot}\right) .
\end{aligned}
$$

Thus the cohomologies at generic point can differ from the cohomologies at the origin. But the Euler characteristic is the same. Summarizing the results we have

Theorem. For any $f^{0}, \chi\left(C_{f^{0}}\right)=\chi\left(C_{0}\right)$ and it is given by (5) .

Now we apply this theorem to the case studied in [5]. Then for the curve (11), which we assume non-singular, of genus $g=\frac{1}{2}(N-1)(N n-2)$ using the results of [5] one finds:

$$
\chi(\Theta)=(-1)^{g-1} N^{N^{2} n-2 N+1}(N n-1)^{N-1}(\Gamma(N))^{2} \prod_{j=1}^{N-1} \frac{\Gamma(j)}{\Gamma(n N+j)}\left(\frac{\Gamma\left(j \frac{n N-1}{N}\right)}{\Gamma\left(\frac{j}{N}\right)}\right)^{2} .
$$

This formula is quite different from what we have for a generic Abelian variety, $\chi(\Theta)=(-1)^{g-1} g$ ! (see [1]).

The formula (8) is still a conjecture since Assumption 1 is not proved in this special case.

\section{$5 \quad$ Examples}

For the hyperelliptic case $N=2$, the formula (8) was proved in [3]. In this section we shall give other examples for which (8) is verified.

Example 1. $g=3$ case: Consider the case $N=4, n=1$ and $X$ is given by $w^{4}+t_{4}(z)=0$, where $t_{4}(z)$ is a polynomial of degree 3. We assume that $t_{4}(z)$ does not have multiple zeros. In this case genus $g$ of $X$ is 3 . The curve $X$ is not hyperelliptic since $w$ has third order poles at infinity and is holomorphic at all other points. Thus $\Theta$ is non-singular and $\chi(\Theta)=6$. Our formula (8) recovers this.

Example 2. $g=4$ case: Consider the case when $N=3, n=2$ and the curve $X$ is given by $w^{3}+t_{3}(z)=0$. The polynomial $t_{3}(z)$ is of degree 5 , we suppose that it does not have multiple zeros. The genus $g$ of $X$ is 4 . In this case the singularity of the theta divisor $\Theta$ consists of one point, say e ( $c f$. [7]). We have the Abel-Jacobi map $\pi$ from the symmetric products of $X$ to $J(X)$. By Riemann's theorem the restriction of $\pi$ to $S^{3}(X)$ gives the surjective map

$$
\pi: S^{3}(X) \longrightarrow \Theta \text {. }
$$

Moreover the isomorphism

$$
S^{3}(X)-\pi^{-1}(e) \simeq \Theta-\{e\}
$$

holds. Since $\pi^{-1}(e) \simeq \mathbb{P}^{1}$, the Euler characteristic of $\Theta$ is given by

$$
\chi(\Theta)=\chi\left(S^{3}(X)\right)-\chi\left(\pi^{-1}(e)\right)+1=-21 .
$$

Our formula (8) precisely recovers this. Notice that the curve $X$ is not hyperelliptic and is not generic among genus 4 non-hyperelliptic curves. 
Those examples support the validity of our assumptions.

For Jacobians of curves satisfying certain genericity conditions, the formula of $\chi(\Theta)$ is given in [8]. For genus 4 case, their formula produces $\chi(\Theta)=-22$. This corresponds to curves whose $\Theta$ has exactly two singular points.

\section{Acknowledgements}

We would like to thank Fu Baohua for comments on the appendix. One of the authors (FS) was partly supported by INTAS grant INTAS OPEN 97-01312.

\section{Appendix A}

We shall give a proof of Proposition 1.

It is obvious that (2) holds if and only if $\left(f_{1}, \cdots, f_{m-g}\right)$ is a regular sequence. We set $I_{j}=A f_{1}+\cdots+A f_{j}$ and, for a subset $T \subset A, Z(T)=\left\{p \in \mathbb{C}^{m} \mid f(p)=0 \quad \forall f \in T\right\}$.

We first notice that $f_{j+1} \notin I_{j}$ for any $j$. Because, otherwise $\operatorname{dim}\left(\mathcal{J}_{0}\right)>g$. Suppose that $\left(f_{1}, \cdots, f_{m-g}\right)$ is not a regular sequence. Then there exist $i$ and $g \in A$ such that

$$
f_{i+1} g \in I_{i}, \quad g \notin I_{i} .
$$

Write $I_{i}$ as an intersection of homogeneous primary ideals $\mathcal{Q}_{j}$ in an irredundant way (cf. [9], Ch IV, §4)

$$
I_{i}=\mathcal{Q}_{1} \cap \cdots \cap \mathcal{Q}_{r} .
$$

This is possible since $f_{j}$ 's are all homogeneous. Then $f_{i+1} \notin \sqrt{\mathcal{Q}_{j}}$ for any $j$. In fact suppose that $f_{i+1} \in \sqrt{\mathcal{Q}_{j_{0}}}$ for some $j_{0}$. Then

$$
Z\left(f_{i+1}\right) \supset Z\left(\sqrt{\mathcal{Q}_{j_{0}}}\right)=Z\left(\mathcal{Q}_{j_{0}}\right)
$$

Notice that

$$
Z\left(I_{i}\right)=Z\left(\mathcal{Q}_{1}\right) \cup \cdots \cup Z\left(\mathcal{Q}_{r}\right)
$$

is the irreducible decomposition of $Z\left(I_{i}\right)$. Since $Z\left(I_{i}\right)$ is the zero set of $i$ polynomials, $\operatorname{dim}\left(Z\left(\mathcal{Q}_{j}\right)\right) \geq m-i$ for any $j$. Then the relation (9) implies that $\operatorname{dim}\left(Z\left(I_{i+1}\right)\right) \geq \operatorname{dim}\left(Z\left(\mathcal{Q}_{j_{0}}\right)\right) \geq m-i$. Since $\mathcal{Q}_{j}$ 's and $f_{j}$ 's are homogeneous, the intersection $Z\left(\mathcal{Q}_{j_{0}}\right) \cap Z\left(f_{i+2}\right) \cap \cdots \cap Z\left(f_{m-g}\right)$ is non-empty. Thus $\operatorname{dim}\left(Z\left(I_{m-g}\right)\right)=\operatorname{dim}\left(\mathcal{J}_{0}\right)>g$, which contradicts the assumption.

On the other hand $g \notin \mathcal{Q}_{j_{0}}$ for some $j_{0}$, since $g \notin I_{i}$. This means that $f_{i+1} g \in \mathcal{Q}_{j_{0}}$ and $g \notin \mathcal{Q}_{j_{0}}$. It follows that $f_{i+1}^{k} \in \mathcal{Q}_{j_{0}}$ for some $k \geq 2$, which is impossible.

\section{References}

[1] A. Nakayashiki and F.A. Smirnov, Cohomologies of Affine Hyperelliptic Jacobi Varieties and Integrable Systems. Comm. Math. Phys. 217 (2001) 623-652

[2] D. Mumford, Tata Lectures on Theta, vol. I and II, Birkhäuser, Boston (1983)

[3] A. Nakayashiki, On the Cohomology of Theta Divisors of Hyperelliptic Jacobians, math.AG/0010006 (2000)

[4] A. Beauville, Jacobiennes des courbes spectrales et systémes hamiltoniens complétement intégrables, Acta Math. 164 (1990) 211

[5] V. Zeitlin, to be published

[6] D. Eisenbud, Commutative Algebra with a View Toward Algebraic Geometry, GTM 150, SpringerVerlag (1994) 
[7] H.M. Farkas and I. Kra, Riemann Surfaces, second edition, GTM 28, Springer-Verlag (1991)

[8] A. Parusiński and P. Pragacz, Chern-Schwartz-MacPherson classes and the Euler characteristic of degeneracy loci and special divisors, J. Amer. Math. Soc. 8 (1995) 793-817

[9] O. Zariski and P. Samuel, Commutative Algebra, Vol. I, second printing, GTM 71, Springer-Verlag (1979) 